\section{Interpreting sequence variants in a clinical context}

To the Editor: I am a medical geneticist whose active practice at the end of my career is devoted almost exclusively to determining the genetic or mutational basis for established, and usually clinically unambiguous, later-onset dominant disorders. Aside from expanded repeat disorders in which the genomic changes are readily interpretable, missense changes in the relevant genes are the next most common category and often present some ambiguity of interpretation. We usually called these mutations on the basis of prior probability, the absence of the change as a known polymorphism in the 1000 Genomes Project, knowledge of the severity/significance of the amino acid change to protein structure (like the mutation prediction programs), and the evolutionary conservation of the amino acid affected. Of this combination of factors, the clinical presentation was usually unavailable to the reference laboratories that we used. This enabled us the override the more conservative interpretation of these laboratories and give useful information to the families who were seeking genetic counseling.

With the expansion of comprehensive genomic databases, we have far more data at our disposal and an enhanced ability to interpret missense gene changes. We also have an increasing rigid and dogmatic set of criteria that are applied by laboratories for the interpretation of missense mutations. These criteria have as their background increasingly prevalent, large-scale whole-exome or -genome studies in which prior probability is too often a smaller factor. If these conservative, risk-averse standards are applied to the patients whom we see with a high prior probability, a large minority-if not a majority-would receive ambiguous results, rendering the study virtually useless. Obtaining family studies or laboratory confirmations are usually beyond the ability of the practicing medical genetics community. We must practice on the basis of very high probability and recognize that there will always be a possibility that the interpretation is erroneous.

With this dilemma in mind, I turned with anticipation to the most recent set of consensus guidelines from the American College of Medical Genetics and Genomics and the Association for Molecular Pathology. ${ }^{1}$ Unlike previous statements that considered only whole-genome sequencing programs, this one at least paid homage to the real world of smaller-scale sequencing of individual genes and gene panels. However, relief was scant and brief. Prior probability and the background frequency of mutation in the individual genes determined from the massive amount of data available were conceded to raise the variant of unknown significance to the very weak, moderate evidence of pathogenicity. This is hardly the level of probability that a practitioner such as myself would need to feel comfortable using in genetic counseling and predictive testing. No effort was made to suggest an algorithm or a statistical calculation by which one could use prior probability, frequency of mutations among the general populations in the databases, and the frequency of the rare disorder among the population to determine a higher degree of probability. When I carried out a simplified exercise with a colleague versed in statistics, we were able to increase the probability that a new missense mutation in a patient who is certain to have a rare autosomal-dominant disorder was overwhelmingly likely to be associated with the disorder and thus serve as a genetic marker in the family. "Moderately likely to be pathogenic" from a genomic perspective became virtually certain from a medical genetics perspective. This was more than certain enough for effective genetic counseling.

We are all aware that counseling in the future will increasingly be done by individuals in specialties other than genetics, who lack the background to add nuance to the prim and technically accurate reports from analytical laboratories. These reports may leave them impotent to carry out the genetic counseling that is required. The geneticist to whom they turn will be better equipped for effective translation of the report but may still be stymied by lack of commitment in the interpretation. Voltaire said 250 years ago, "the perfect is the enemy of the good." The perfect reports that we get are certainly the enemy of effective genetic services. The College has the ability to mitigate this and must try to do so.

\section{DISCLOSURE}

The author declares no conflict of interest.

Stephen Cederbaum, $M D$

Division of Genetics, Departments of Psychiatry, Pediatrics and Human Genetics, University of California Los Angeles, Los Angeles, California, USA. Correspondence: Stephen Cederbaum (scederbaum@mednet.ucla.edu)

\section{REFERENCE}

1. Richards S, Aziz N, Bale S, et al. Standards and guidelines for the interpretation of sequence variants: a joint consensus recommendation of the American College of Medical Genetics and Genomics and the Association for Molecular Pathology. Genet Med 2015;17:405-424.

Advance online publication 5 November 2015. doi:10.1038/gim.2015.150 